be it remembered, because we cure the disease, but because we get rid, for the time, of an obstructing secondary evil, and are hence permitted an opportunity of combatting the primary malady by such measures as we deem fitting.

I now come to the intention of the operation proposed by Dr. Hastings, and executed by his friend-an intention which differs essentially from that proposed in two of the cases before named. In them, fluid is sought to be withdrawn from the cavity of the chest; in this, from a cavity situated in the substance of the lung itself, with a view of removing air which distends it, and prevents the approximation, and, consequently, the adhesion, of its sides.

The first serious practical difficulty which here presents itself is that of diagnosis; for it is obvious that, unless the parietes of the cavity adhere firmly to the walls of the chest, great hazard must arise from rashly penetrating to its interior; and yet it appears to me that the most dextrous stethoscopist or percussor cannot, with precision, determine the existence of an adherent cavity; to ascertain the presence of a cavity even is not quite so easy as is often supposed; but here we have not only to do this, but also to ascertain that such excavation is throughout adherent. On such grounds alone the operation is one which should not be rashly undertaken.

But I will suppose a case-and such appears to be that of Dr. Hastings - in which the diagnosis was perfect-where the walls of the cavity did adhere to those of the chest, its interior containing air only. If the operation be ever admissible, it is in such a case; but even then I should be disposed to question the principle on which it rests. A few words will explain my meaning.

The cavity is presumed to contain air, admitted, of course, through the trachea; this distends it, and renders approximation of its sides impossible. To remove this, a new opening is artificially made into its interior, with the expectation that air therein contained will be finally evacuated.

When this theory is examined on physical principles, its fallacy seems to me strongly marked. All know that the atmosphere presses with an average weight of fifteen pounds on each square inch of surface, and that it is by the influence of such pressure that air permeates the lungs, when, by an inspiratory muscular effort, their containing cavity is angmented in capacity. Now, the very same amount of pressure which, acting through the windpipe, distends the Iungs, or the abnormal excavation with air, equally acts through an opening artificially made, and the result is, that the alternation of ingress and emission connected with the antagonistic acts of inspiration and expiration, go on as in the natural state, the only difference being. that according to the relative area of the external opening, as contrasted with the glottis, so will be the amount of air which enters by them respectively.

If I could imagine that any great advantage is attainable in phthisis by approximating the sides of a tuberculous cavity, I should be disposed to think that the plan long ago proposed by Dr. Ramadge, and referred to by Dr. Herbert. in your number fo: the 18th of January last, was the more likely to succeed. By forcibly distending such portions of the lung as are still in a normal state, it is not impossible that a pressure might be exerted on the cavity adequate to counterbalance the atmospheric pressure which distends it, thus placing its internal surfaces in closer vicinity, at least, if not in contact. But I much fear that the plan is practically but seldom crowned with success; at all events, it has fallen to my lot to see numerous cases which had been treated by the forcible inspiration suggested by $\mathrm{Dr}$. Ramadge, and in no instance did any good results follow. I am bound, however, to say, that all were cases of consumption very far advanced; cases in which small hope of success under any method of treatment could have been rationally indulged.

Your correspondent, Dr. Herbert, who appears as the advocate of Dr. Ramadge's practice, and an opponent of Dr. Hastings's operation, states that within the last " eighteen months" he has been present at " seven such operations performed under Dr. Ramadge's directions." As these words occur when he is commenting on the undue severity used in the operation immediately under review, a little obseurity arises as to whether Dr. Ramadge's cases involved the opening of tuberculous cavities, or simply puncture of the chest, with other intentions. It would be satisfactory to have this obscurity removed, and to know, if of the first kind, what were the results.

But admitting the expediency of operating in certain cases, one thing at least is clear, that even by success we can only hope to get rid of a secondary, though, without doubt, important complication. Persons who die of phthisis do not die because they have one or more empty cavities in the lungs. We appear now to have pretty certain evidence of the fact, that in numerous instances-either from some unknown change in the constitution, dependent on natural causes, or possibly from the influence of remedial means employed - tuberculous cavities often tend to cicatrize; and, provided always that the diathesis is removed, and the formation of new cavities consequently prevented, there appears to me reasonable evidence that consumption is, in one or other way, not unfrequently cured; using that term in a restricted sense.

Locally considered, phthisis is a progressive malady, and men seem now generally agreed that it originates in some derangement of the assimilative function, which reacts on the lungs, and there lays the foundation of that condition with which its more prominent symptoms are connected; but we know that a very large amount of the lungs may be rendered useless by other causes, without this leading to a fatal issue, and we hence legitimately may conclude, that to counteract at its source the constitutional derangement on which the local affection depends, is the proper object of inquiry with all who take an interest in this most fatal and yet most interesting disease.

Whether such an end is best attained by naphtha, hy alkalies, by climate, counter-irritation, or a score of other plans hitherto advanced, it remains for future investigation to determine; but of one thing we may, I think, be pretty well conviaced, that the healing of an excavation, even when accomplished, is not a cure of the malady, and I consecuently object to Dr. Hastings's operation, not only because it is in itself hazardous and uncertain, but because, in its best aspect, it deals with an effect, and not a cause. March 17 th, 1845 .

\section{A NEW MODE OF APPLYING LIGATURES TO NAVI.}

By J. C. Christophers, Esq. Surgeon, London.

THE written description of any mechanical performance necessarily makes it appear more or less intricate; nor do diagrams, wherein the tying of knots is depicted, go far to illustrate the same; but any one once witnessiug the operation I am about to describe, (performed on a piece of cloth,) would be able, I feel confident, to apply it on the living body.

The operation itself is very simple, requiring only a needle and a piece of waxed silk for its performance; it is very easily executed, and may be divided into tno stages-1st, That in which the ligature is passed; 2ndly, That in which the ligature is tightened; and may be described as follows:-

1st. Take a piece of strong silk, or a ligature, well waxed, and three quarters of a yard in length ; thread a curved needle with the same, leaving the ends equal,* and pass it, double, under the centre of the part required to be removed; this dnne, cut the ligature in the middle, leaving the needle attached to the inferior portion; take the same, and pass it through the skin immediately below the part to be strangulated. Thread the needle with the superior portion of the ligature, and pass it throuoh the skin in an opposite direction, immediately above the part to be strangulated. Remore the needle.

2 ndly. Tie as tightly as possible the two erids of the loop that includes the inferior half of the tumour, and strangrulate that portion of the part to be removed. 'Tie, in the same manner, the other two ends of the loop, including the superior half of the tumour, and strangulate that portion of the part to be removed. The four ends of the ligature remaining are now to ba tied tightly ard alternately the one to the other, and the operation is completed. The whole mass to be removed being thus entirely enclosed in a double circle by the ligature both from within and without, must be effectually strangulated.

The difficulties attending the following case, led to the invention of the method described:-

Case.-The child of Mrs. G-, residing in Henrietta street aged three months, presented a nævus, about the size of a nut, situated directly opposite to, and closely bordering on, the internal canthus of the right eye, extending thence upwards towards the eyebrow; and inwards towards, and reposing on, the nose, reaching nearly to the median line; it was very florid, and about to ulcerate.

One glance at the situation of this tumour will show how inapplicable the methods usually adopted would have been. The child was not weaned, therefore the method by needles would have been most inconvenient both to the mother and to the infant, to say nothing of the difficulty to apply them in that situation. Excision would have been no less so, as the hæmorrhage would have been considerable, and in a situation most difficult to control; the vicinity to the eye would greatly impede the necessary compression. To obviate these inconveniences, I

* It facilitates the application to dye one-half of the ligature in ink, the more readily to distinguish the ends after it is divided. 
devised and-assisted by my friend Mr. Lonsdale-performed the operation described. No blood was lost; the performance occupied perhaps two minutes, and might be accomplished in less time. On tightening the ligature, the nævus became perfectly white, and so violent was the constriction, that the secretion from the mucous follicles was forced out all over its surface. The child did not appear to suffer much during the operation, and ceased to cry or to exhibit any symptom of pain five minutes after its completion, and immediately went to sleep.

The following day, the child appeared quite well and cheerful; had passed an excellent night; the line of separation very marked, extending one-eighth of an inch all round the part constricted, which was half its former size; no swelling of the face or nose, nor cedema of the eyelid. All went on well, and on the eighth day the slough with the ligature was thrown off, leaving a circular wound of considerably less dimensions than the nævus presented prior to the operation.

The advantages this method appears to possess over those in general use, may be stated as follows:-

1. That it requires no other apparatus than a needle and a piece of silk; and that it is very easily, quickly, and safely performed.

2. That it leaves less deformity than the operation by excision, or by the common mode of applying ligatures, the sound skin being drawn around the base of the nævus, and so held, as by two points of suture, almost to fill the space previously occupied by the same.

3. That neither needle nor other apparatus is left after the operation is completed, with risk of injury to other parts, nor to the inconvenience of the mother, the infant, or the nurse.

4. That it can be applied easily in situations where other means cannot be used conveniently, at any depth, or however superficial, to a flat as readily as to a raised surface, without risk of hæmorrhage at the time of operating, or subsequently thereto.

5. That the ligature passing beneath and completely encircling the parts to be removed, the strangulation must be perfect, and that it can be seen to be so at the period of operating, thereby putting aside all doubt as to the result.

Upper Montague-street, February 14th, 1845.

\section{ON THE POISONOUS EFFECTS OF SAVIN, (JUNIPERUS SABINA.)}

By H. Letheby, Esq. M.B., Lecturer on Chemistry at the London Hospital.

A youxG woman, M. A. N-, aged 21 , had enjoyed remarkably good health up to twelve hours before her death. On the night of 'Tuesday, April the 22nd, she supped with her lorer, by whom she was far advanced in the family way. She was then cheerful and hearty, but about three o'clock in the morning, she awoke her mother, with whom she slept, and complained of a violent pain in her stomach. She was very sick, and her mother, believ. ing that it arose from a too hearty meal, got up and gave her some brandy and water. The sickness, however, was not diminished, and she subsequently became insensible. About eight o'clock, her mother sent for the parish surgeon, whose assistant eame to see her; he prescribed some ordinary medicines, which, from her insensibility, they were unable to administer. Fearing that there might be some danger, the mother sent for another surgeon, Mr. Newth, who got to her about eleven o'clock. She was lying on her back, perfectly insensible, breathing laboriously, and with.stertor; she was foaming at the mouth, her countenance was turgid, the eyes shut, with the pupils much contracted; her limbs were also convulsed, and the mother told him that she thought she had been suddenly taken in labour. He therefore made an examination per vaginam, but found that the os uteri was only slightly dilated; the membranes, however, were ruptured, and the uterus was still acting vigorously, each contraction being accompanied by a convulsive tremor. Believing it to be a case of puerperal convulsions, he bled her, and applied cold to the head, but this did not in any degree restore her. As the os uteri became more fully dilated, he found that the head of the child presented, and that it was gradually descending. About three o'clock, while the labour was thus progressing, she uttered a low moan, and in a few minutes ceased to breathe. Mr. Newth immediately sent for the forceps, and delivered her of a male child, which was dead; as far as he could judge from its appearance, and the mother's report, deceased was somewhere between the term of the seventh and eighth month of pregnancy.

Twenty-four hours after death, Mr. Newth made an examination of her body. It bore no marks of violence, was well formed, and rather robust. On opening the head, he found that the vessels upon the surface of the brain were much gorged with black fluid blood, and in proceeding with sections of the cerebral substance, discovered that the grey matter was infiltrated in various parts by the same dark-looking blood; this he especially noticed in the anterior part of the corpus striatum. The choroid plexus was also gorged; the ventricles were empty. The lungs were a little congested on the dependent parts, but otherwise healthy. He did not examine the heart, nor did be pay attention to any of the abdominal viscera besides the stomach. This on being opened appeared rather paler than usual, excepting one or two spots, which were red, as if blood had been effused into the mucous tissue. It contained about four ounces of fluid, which he secured, and brought to me for examination. This fluid had a dark brownish-green colour, smelt of digesting matter, and exhibited an acid reaction. A part of it was subjected to distillation in a retort. The portion which came over was turbid and opaque ; it smelt and tasted like oil of savin. When examined by the microscope, it was found that the turbidity was due to minute oil globules. The whole was therefore shaken with ether, which instantly rendered it transparent; and on allowing this to stand and evaporate spontaneously, I procured several small drops of a yellow oil, having all the physical character of oil of savin. A little of the sediment from the original bottle was afterwards examined under the microseope, and compared with the powder of the dried plant; their structure appeared to be perfectly identical. The remainder of the fluid was filtered, and the residne digested with ether, by which means there was obtained a green solution, containing resin and the vegetable colouring matter, chlorophylle. In order, however, to be certain of all results which were thus manifested, I insiituted a parallel series of experiments with a little powdered savin, and there could be no doubt in my mind of their identity. It afterwards occurred to me that porter might possibly yield similar products. I therefore distilled four ounces of this fluid, and after rejecting the alcoholic portion which first came over, I obtained a liquid which was slightly turbid, from the presence of oil; but if we except this particular there was no similitude whatever between the results.

Remarks. - In the evidence which was given before the coroner (for I was unable to attend the inquest) an opinion was entertained that deceased had died of puerperal convulsions, and a verdict to that effect was accordingly returned. It appears, however, after carefully reviewing the case, and making a comparison of the symptoms with those which are produced by savin upon the lower animals, that this poison might have been the cause of her death, for one of the most constant effects produced by it is a great congestion of the venous and capillary systems, together with profound coma. For instance, to take one case out of many. Two drachms of powdered savin, which had been kept for some years, was administered with food to a small terrier; in about one hour the animal appeared a little dull and sleepy, but it was otherwise unaffected, and even this appearance might have escaped notice, if we had not been watching for effects. In another hour and a half it began to vomit, bringing up the greater part of its food, and some of the poison. This vomiting was repeated at varions intervals for about one hour and a half. It then appeared exhausted, and laid down, as it were, to sleep, but it never again rose, for in two hours it was found in the same position, perfectly insensible, and comatose, breathing with a peculiar puffing noise. The eyes remained closed, the pupils were contracted, the limbs moved when they were pinched, and the heart was beating with a slow, labouring action. It lay in this position for about eight hours, during which time a great deal of blood was passed by stool, and there was an occasional convulsive movement of the hind legs. Its breathing became slower and slower, until death took place, which happened fourteen hours after the administration of the poison.

Twenty-four hours after death the body was found in the same position in which the animal had lain down some hours before it died. The vessels on the surface of the brain were much congested and filled with dark blood; the cboroid plexus was also gorged, and the substance of the brain more turgid than natural. The lungs were collapsed, and of a bright scarlet colour; the right cavities of the heart were full, the left empty. The liver was almost black, the gall-bladder full of very green bile. The spleen purplish black. The stomach was pale externally, and so was the upper part of the small intestines; the lower, however, were exceedingly congested, and looked as if blood had been effused between the muscular strix. On opening them, they were found to be highly vascular, and covered with a layer of sanguineous fluid. The stomach contained two ounces of a darkbrownish liquid, which gave a tarbid fluid on disti'lation, from which a little savin oil was obtained. The internal coat of the stomach was pale, excepting in two or three spots, where the mucous coat was injected with blood.

Now, it will be obserzed, that these symptoms and appearances 